\title{
DELAMINATION VISUALIZATION IN CFRP LAMINATED CONCRETE STRUCTURES USING PULSED LASER-BASED ULTRASONIC PROPAGATION IMAGES
}

\author{
CHANGGIL LEE ${ }^{*}$ AND SEUNGHEE PARK ${ }^{\dagger}$ \\ Sungkyunkwan University \\ Suwon, Korea, Republic of \\ e-mail: tolck81@gmail.com \\ † Sungkyunkwan University \\ Suwon, Korea, Republic of \\ e-mail: shparkpc@skku.edu
}

Key words: Pulsed laser scanning, Ultrasonic waves, carbon-fiber-reinforced polymer (CFRP) delamination, Concrete structures, Flaw imaging, Wavenumber filtering

\begin{abstract}
One of the most critical characteristics of concrete structures is the brittleness of the concrete and hence the concrete is vulnerable to tension force. To overcome this drawback, the use of carbon-fiber-reinforced polymer (CFRP) sheets and/or plates laminated on the surface of concrete structures has increased. However, the efficiency of the CFRP reinforcement to endure the tension force is reduced when the CFRP sheets and/or plates are delaminated from the concrete structures due to external loads and impacts during service. This study presents the application of a pulsed laser scanned ultrasonic wave propagation imaging technique for detecting delamination damage of the CFRP laminated concrete structures. For the experimental studies, the delamination was artificially formed when the CFRP sheets were attached on the concrete structures and scanned using the Nd: YAG pulsed laser scanning system. By applying a frequency-wavenumber filtering algorithm to the wave responses measured from the Nd: YAG pulsed laser scanning system, flaw images of the delamination of the CFRP sheets were successfully obtained. This result confirmed the feasibility of the pulsed laser scanned ultrasonic wave propagation imaging technique to serve as an effective structural health monitoring system for actual CFRP laminated concrete structures.
\end{abstract}

\section{INTRODUCTION}

One of the most critical characteristics of concrete structures is the brittleness of the concrete and hence the concrete is vulnerable to tension force. To overcome this drawback, carbon-fiber-reinforced polymer (CFRP) sheets and/or plates have been laminated on the surface of concrete structures. The efficiency of the CFRP reinforcement to endure the tension force is reduced when the CFRP sheets and/or plates are delaminated or de-bonded from the concrete structures due to environmental influences such as external loading, impacts, shrinkage due to the temperature differences, and so on. Therefore, the delamination damage of CFRP sheets and/or plates needs to be effectively detected. For effective damage detection, we applied a damage visualization method using a pulsed laser scanning system.

In many types of actual structures, inspecting the structural health conditions is extremely difficult because of the limited accessibility of the structures. To overcome 
this drawback, the development of remote non-contact structural health monitoring/nondestructive testing (SHM/NDT) technologies is urgently needed. Additionally, reliable SHM/NDT methods must be intuitive, so that inspection results can be easily understood, and must have high throughput [1]. To tackle this issue, many studies based on acoustic and ultrasonic technologies have been proposed using a laser interferometry, a laser vibrometer, and a pulsed laser etc., because the ultrasonic waves are very sensitive to the mechanical properties of structures. The holography based imaging technique, one of the full-field ultrasonic wave imaging techniques, requires a highly diffusive target surface. Also, holographic images can be obtained clearly in dark environments and hence this method is not suitable for the general purpose of SHM/NDT, although it has non-contact inspection capability [2]. Dynamic responses can be collected by laser Doppler vibrometry (LDV) which measures the vibrational velocity of target structures. Although the performance of LDV has been improved in terms of scan angles and automatic focusing [3], it still has a disadvantage, whereby retroreflective film needs to be attached to the target surfaces to improve the signal-to-noise ratio (SNR) when a scanning head is located far from the target [4-6]. In contrast, laser interferometry, which also senses the vibration responses of target structures, works well without the retroreflective film; however, its performance has not been demonstrated when the distance between the scanning head and target surface is greater than $0.5 \mathrm{~m}[7,8]$. To overcome these drawbacks, a laser beam pulse can be used to generate ultrasonic waves. The laser beam pulse can provide many advantages including fast wave generation with low pulse energy, high spatial resolution, inspection ability on a complex target, and so on [1].

In this study, ultrasonic wave propagation images were obtained using the pulsed laser scanning system and flaw images of the CFRP laminated concrete specimens were then visualized based on the characteristics of the standing waves at the delamination damage.
The feasibility of the proposed method was verified by a series of lab-scale experiments.

\section{ULTRASONIC WAVE PROPAGATION IMAGING (UWPI) PROCESS}

An ultrasonic wave propagation imaging (UWPI) system consists of a Q-switched laser system, a laser mirror scanner based on a galvanometer, an ultrasonic sensor, a highspeed digitizer, and an image processor that was re-arranged based on information in a previous work [1]. A Q-switched Nd: YAG diode-pumped solid-state laser is used for the pulsed laser system, which has the wave length of $532 \mathrm{~nm}$ and maximum pulse repetition frequency of $20 \mathrm{~Hz}$ [9]. The pulsed laser beam impacts a specific point on a target structure by the laser mirror scanner which is designed so that two galvanometers are operated at the wave length of $532 \mathrm{~nm}$. The operating angles of the galvanometers are orthogonal to each other so that the laser beam can scan a twodimensional (2D) area rapidly. Additionally, the maximum angular velocity of the galvanometer is 100 rad/s within the range of $0.35 \mathrm{rad}$. The laser beam reflected at the laser mirror scanner is focused on the target structure with an f-theta lens which is installed at the end of the laser mirror scanner. In this study, the f-theta lens is designed so that the distance between the laser mirror scanner and the target structure is $2 \mathrm{~m}$. The focused laser beams scan the target structure vertically upward, step horizontally, and then scan vertically downward. Therefore, the scanning laser beams generate a grid of the laser impingement points and the pitch of the grid can be adjusted by the control algorithm of the laser system.

When the pulsed laser beams impact the target structure, ultrasonic waves are generated by the thermo-elastic mechanism and are then propagated. The multiple wave responses can be measured by only a single PZT attached to the rear side or the front side of the structure. The measured time signals are placed at each laser impinging point and the UWPI is obtained. The wave propagation is then 
visualized in 3 steps. i) Measuring and filtering the signals; to improve the SNR, a pass-band filter can be applied. ii) Placing the processed signals on the laser impinging points; the signal group results in a three-dimensional (3D) space with respect to the horizontal, vertical, and time axes. iii) Slicing the 3D space along the time axis; finally, the wave propagation image can be obtained by slicing repeatedly within the measuring period [1].

\section{PROCESS FOR DAMAGE VISUALIZATION}

The flaw images can be easily obtained by observing the standing wave at the delaminated area after eliminating the strong forward wave. To filter out the forward wave, the wavenumber filtering concept is applied in this study. First, the wave propagation image is transformed from the time/space domain to the frequency/wavenumber domain by 3D Fourier transform [2]. The time signals are primarily filtered by a bandpass filter to increase the SNR.

The forward wave can be filtered out by eliminating the positive or negative side of the frequency/wavenumber domain signals using the window function as shown in Equation (1) because the wavenumbers represent the propagating direction of the waves. If a structure contains a flaw, the forward waves are scattered on the delaminated area and the backward portions of the waves including reflections appear. The forward or backward portions of the waves due to damage can be extracted by the wavenumber filtering.

$$
\bar{W}\left(k_{x}, k_{y}, \omega\right)=W\left(k_{x}, k_{y}, \omega\right) M\left(k_{x}, k_{y}, \omega\right)
$$

where $\bar{W}\left(k_{x}, k_{y}, \omega\right)$ is the wavenumber filtered data, $W\left(k_{x}, k_{y}, \omega\right)$ is the UWPI data, and $M\left(k_{x}, k_{y}, \omega\right)$ is the window function for wavenumber filtering, which are on the wavenumber and frequency domain. In this study, a rectangular function is used for the window function. The filtered UWPI data is inversely transformed from the wavenumber and frequency domain to the spatial and time domain.

If the forward wave encounters a discontinuity point due to damage, some portions are transmitted and others are reflected. Additionally, a few portions of the waves stand at the discontinuity point. All of the propagating waves at the tip of the discontinuity point can be expressed with an assumption of two harmonic waves propagating in the forward and backward directions [10]. A term on the standing wave can be easily derived by simple trigonometric function. However, the terms on the forward and backward waves are coupled when the term on the standing wave appears. Therefore, it is difficult to directly extract the term on the standing wave.

To easily extract the standing wave portion, the energy of the total waves is used. The energy of all the waves comprises the forward and backward wave energies and the standing wave energy. In the total wave energy, the forward and backward waves retain their forms with the additional term due to the standing wave energy and hence the only standing wave energy can be simply extracted by subtracting the forward and backward wave energies from the total wave energy [11]. The damage can then be identified by extracting the standing wave energy. The standing wave due to the damage is extracted by subtracting the transmitted and reflected portions at the damage from the total waves.

Finally, the damage can be visualized by calculating the root mean square (RMS) values using the filtered signals as shown in Equation (2) [12]. Because the ultrasonic waves are reflected from the damage, distribution of the energy is changed. These changes are represented as changes in the RMS values and could be used for damage detection.

$$
\bar{w}_{S}^{r m s}(x, y)=\left[\frac{1}{N} \sum_{i=1}^{N}\left\{\bar{w}_{S}(x, y, t)\right\}^{2}\right]^{1 / 2}
$$

where $\overline{w_{s}}$ is extracted standing wave portions in the time and space domains. 


\section{EXPERIMENTAL STUDY}

\subsection{Experimental setup}

In this study, unidirectional CFRP sheets were used for the reinforcement of concrete specimens. The strength of the concrete specimens was about $30 \mathrm{MPa}$. In this study, the strength of the concrete was not important because the purpose of this study was to detect the delamination between the CFRP sheets on the concrete. The material properties of the CFRP sheet are described in Table 1. We laminated 6 sheets with epoxy glue on the concrete specimens in the same direction as that of the fiber ply and small pieces of paper wrapped with polyvinyl were inserted between the sheets at specific points so that the delamination could be simulated. An acoustic emission sensor with an embedded amplifier was used to measure the responses of the laserinduced ultrasonic waves. In the frequency range between $100 \mathrm{kHz}$ and $500 \mathrm{kHz}$, the sensitivity of the sensor is relatively high. Specifically, the maximum sensitivity of the sensor is about $120 \mathrm{~dB}$ at the frequency of 200 $\mathrm{kHz}$. Therefore, the measured time signals were filtered using a digital bandpass filter with the frequency range between $100 \mathrm{kHz}$ and $500 \mathrm{kHz}$ to eliminate high-frequency noise.

Table 1: Material properties of the CFRP sheets

\begin{tabular}{c|c}
\hline Properties & Values \\
\hline $\begin{array}{c}\text { Thickness (mm) } \\
\text { Tensile strength } \\
\text { (Kgf/cm*width) } \\
\begin{array}{c}\text { Young’s modulus (Tensile) } \\
\text { (Kgf/cm*width) }\end{array}\end{array}$ & 0.111 \\
\hline
\end{tabular}

The concrete specimens laminated with the CFRP sheets are shown in Figure 1. For the practical purpose, the direction of the fiber ply of the CFRP sheets is longitudinal to the specimen so that the tensile side of the concrete can be unidirectionally reinforced. The dimensions of the test specimens are described in Figure 1. First, an intact specimen was scanned to obtain reference data. The scanning areas and the sensor locations are shown in Figures 2 and 3. Then, two types of delamination damage were artificially formed as described in Figures 2 and 3. The first type of damage was induced by simply placing the paper between the two CFRP sheets (hereafter, Type I). For the second type of delamination, we cut out small pieces of the CFRP sheet in the third layer and replaced the cut out pieces with the paper (hereafter, Type II). Type I damage was applied to the case with varying damage location and Type II damage was applied to the case with varying damage size.

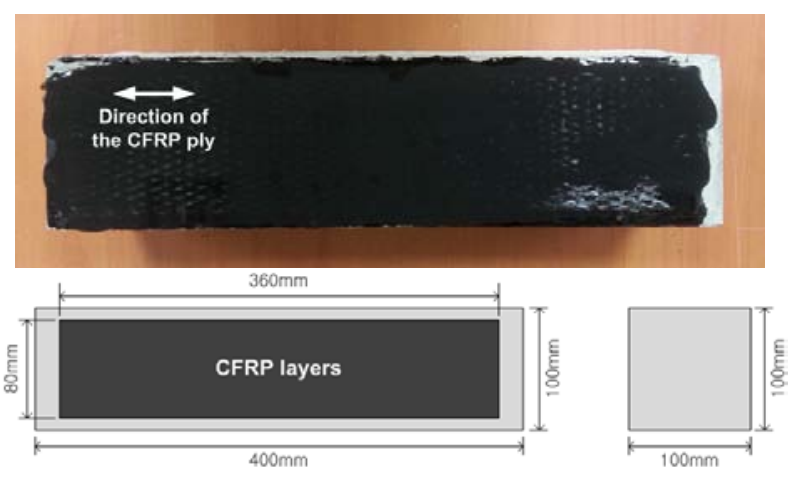

Figure 1: Configuration of specimens

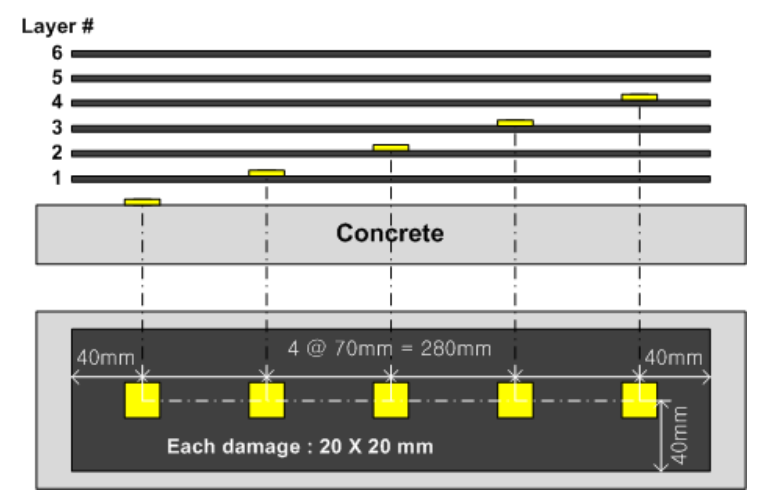

(a) Scheme of damage case I

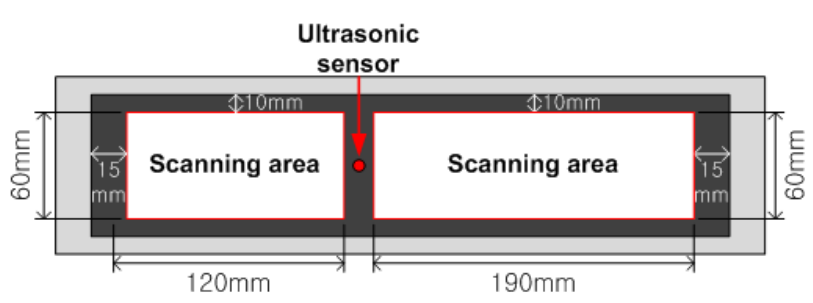

(b) Scanning area and sensor location

Figure 2: Case I: Variation in the damage location 


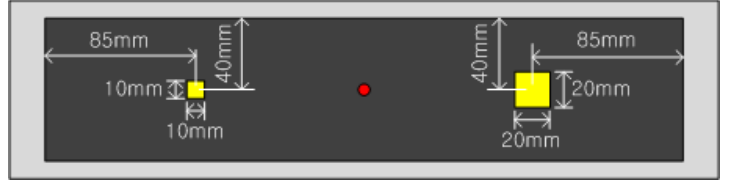

(a) Scheme of damage case II

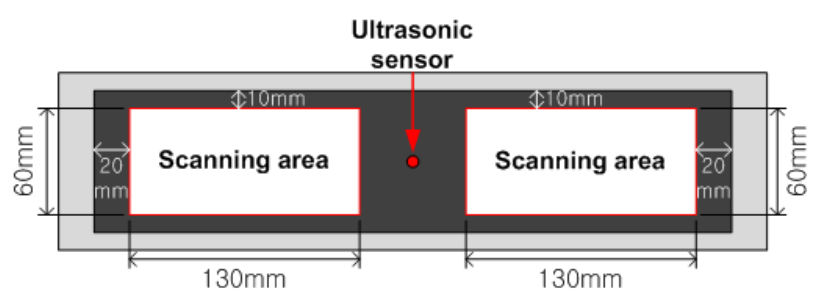

(b) Scanning area and sensor location

Figure 3: Case II: Variation in the damage size

\subsection{Results and discussion}

As described in the previous subsection, three concrete specimens with different conditions were scanned using the pulsed laser scanner and the flaw images are shown in Figure 4. First, some lines and points, which were induced by the direction of the CFRP ply of the top layer, could be observed in the case of intact condition, although the CFRP layers were fully bonded. This might be due to the spatial resolution of the flaw image because the width of the ply was wider than the scanning interval of $1 \mathrm{~mm}$ in this study. On the other hand, all of the flaws due to delamination damage are observed clearly in the case of the variation in delamination location. All of the flaws due to delamination damage were the same size at the stage of experimental setup and the visualized flaws also show the same size regardless of the damage location, as shown in Figure 4(b). Unfortunately, one of the flaws due to damage is not clearly observed in the case of Type II, as shown in Figure 4(c). In this case, the standing waves were dissipated rapidly when the damage increased in size because the medium at the delamination point for the wave propagation was cut out and hence the waves could not stand, so that the energy of the standing waves could not be condensed sufficiently. Therefore, the small flaw $(10 \mathrm{~mm}$ $\mathrm{X} 10 \mathrm{~mm}$ ) is clearly visualized while the large flaw (20mm X 20mm) is weakly estimated. In terms of the practical issues, the proposed methodology is much more reliable when the small sized (less than $20 \mathrm{~mm} \quad X \quad 20 \mathrm{~mm}$ ) delaminations are detected; hence, this result confirms that the proposed SHM/NDE system is suitable for detecting the delamination damage of the CFRP laminated concrete structures.

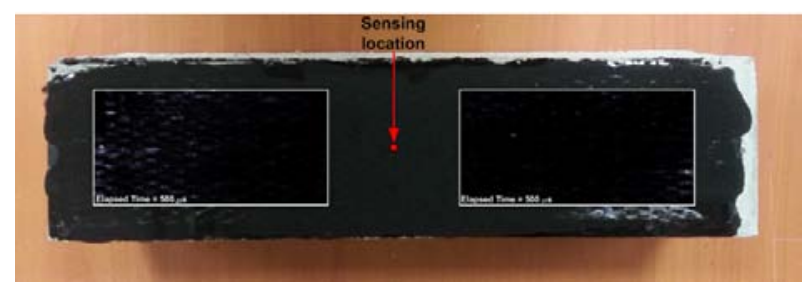

(a) Intact condition

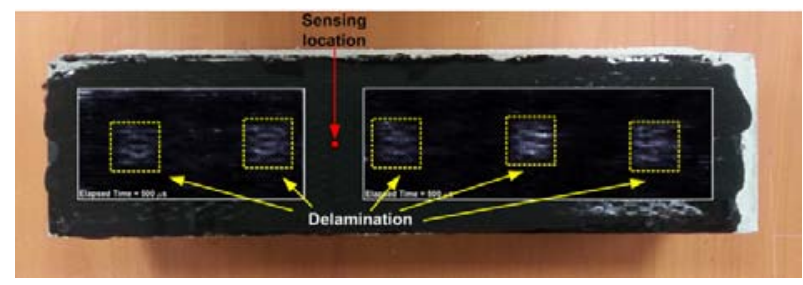

(b) Damage case I

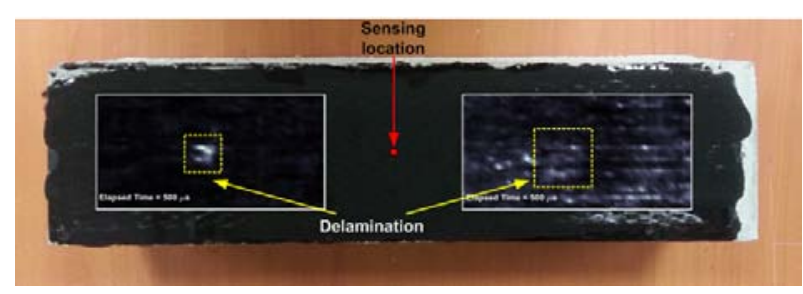

(c) Damage case II

Figure 4: Comparison among three different flaw images

\section{CONCLUSIONS}

In this study, we considered visualization of the artificially formed delamination damage of CFRP laminated concrete with different damage conditions. Two damage condition cases were considered: the variation in the damage location and the variation in the damage size. In the case of investigating different damage locations, all of the delamination damages (20mm X 20mm) were clearly visualized regardless of the different damage locations. However, in the case of investigating different damage sizes, the smaller sized flaw due to damage $(10 \mathrm{~mm} X$ $10 \mathrm{~mm}$ ) was more clearly detected than the 
larger sized flaw due to damage $(20 \mathrm{~mm} \mathrm{X}$ $20 \mathrm{~mm}$ ). Because, the delamination was formed by cutting small parts of the CFRP sheet and by replacing it with other material, the energy of the standing waves due to the delamination dissipated rapidly for the larger sized damage case compared to the smaller sized damage case.

As a result, delamination damage with the size of $20 \mathrm{~mm} \mathrm{X} 20 \mathrm{~mm}$ could be be very clearly identified at even multiple locations. However, this does not mean that the damage size of $20 \mathrm{~mm}$ X $20 \mathrm{~mm}$ is the limit of detection capability of the proposed method and the upper limit and lower limit of the size of the flaw due to damage needs to be found for practical use.

This study was performed to verify that the delamination damage between the CFRP sheets, which were attached to the concrete specimens, could be visualized using the pulsed laser scanning system. This method needs to be performed under more various conditions to test its validity in application to actual structures.

\section{ACKNOWLEGEMENTS}

This research was supported by grant from the National Research Foundation of Korea (NRF) funded by the Korea government (MSIP) (NRF-2014R1A2A1A11054299) and a grant from the Construction Technology Research Program (14SCIP-B088624-01) funded by the Korea government (MLIT).

\section{REFERENCES}

[1] Lee, J.-R., Chia, C.C., Shin, H.J., Park, C.-Y., and Yoon, D.J. 2011. Laser ultrasonic propagation imaging method in the frequency domain based on wavelet transformation. Optics and Lasers in Engineering 49:167-175.

[2] Green, Jr. R.E. 2004. Non-contact Ultrasonic Techniques. Ultrasonics 42:916.
[3] Polytech. 2012. PSV-500 Scanning Vibrometer Datasheet, http://www.polytec .com/fileadmin/user_uploads/Products/Vi brometers/PSV-500/documents/OM_DS_ PSV-500_2012_09_3000_E.pdf

[4] Köhler, B. 2006. Dispersion relations in plate structures studied with a scanning laser vibrometer. Proc. of the ECNDT 2006, Paper Mo. 2. 1. 4, 1-11.

[5] Muhammad, N.S.B., Hayashi, T., Murase, M., and Kamiya, S. 2009. Analysis of guided wave propagation by visualizing in-plane and out-of-plane modes. AIP Conference Proceedings, 1096: 774-781.

[6] Castellini, P., Martarelli, M. and Tomasini, E.P. 2006. Laser Doppler vibrometry: development of advanced solutions answering to technology's needs. Mechanical Systems and Signal Processing 20: 1265-1285.

[7] Pouet, B., Breugnot, S., and Clémenceau, P. 2005. An innovative interferometer for industrial laser ultrasonic inspection. AIP Conference Proceedings 760: 273-280.

[8] Blum, T., Pouet, B., Breugnot, S., and Clémenceau, P. 2008. Non-destructive testing using multi-channel randomquadrature interferometer. AIP Conference Proceedings 975: 239-246.

[9] Quantel. 2012. CFR Specification, http://www.quantellaser.com/tl_files/clien t/MY\%20QUANTEL\%20SPACE/Sales\% 20Literature/CFR_Specs_EN_072012.pdf (2012, accessed 26 October, 2014)

[10]Silk, M.G., and Bainton, K.F. 1979. The propagation in metal tubing of ultrasonic wave modes equivalent to Lamb waves. Ultrasonics 17:11-19.

[11]An, Y.-K., Park, B., and Sohn, H. 2013. Complete noncontact laser ultrasonic imaging for automated crack visualization in a plate. Smart Materials and Structures 22: 025022(10pp).

[12]Radzieński, M., Doliński, Ł., Krawczuk, M., Żak, A., and Ostachowicz, W. 2011. Application of RMS for Damage Detection by Guided Elastic Waves. Journal of Physics: Conference Series 305: art. no. 012085, 1-11. 